JOURNAL OF SYNCHROTRON RADIATION

ISSN 1600-5775

Received 9 July 2019

Accepted 23 September 2019

Edited by P. A. Pianetta, SLAC National Accelerator Laboratory, USA

Keywords: diffraction-limited storage ring; energy spread; cross-spectral density function; coherent-mode representation; degree of coherence; coherent fraction.

Supporting information: this article has supporting information at journals.iucr.org/s
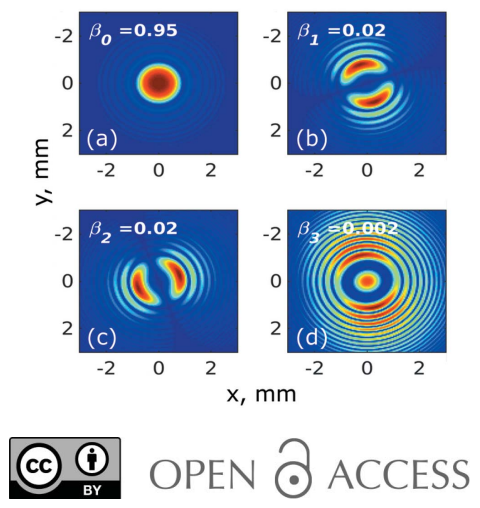

\section{Coherence properties of the high-energy fourth-generation X-ray synchrotron sources}

\author{
R. Khubbutdinov, ${ }^{a, b}$ A. P. Menushenkov ${ }^{b}$ and I. A. Vartanyants ${ }^{a, b *}$

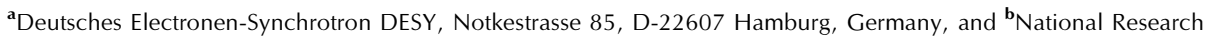 \\ Nuclear University MEPhI (Moscow Engineering Physics Institute), Kashirskoe shosse 31, Moscow 115409, Russian \\ Federation. *Correspondence e-mail: ivan.vartaniants@desy.de
}

An analysis of the coherence properties of the fourth-generation high-energy storage rings with emittance values of $10 \mathrm{pm}$ rad is performed. It is presently expected that a storage ring with these low emittance values will reach diffraction limit at hard X-rays. Simulations of coherence properties were performed with the $X R T$ software and an analytical approach for different photon energies from $500 \mathrm{eV}$ to $50 \mathrm{keV}$. It was demonstrated that a minimum photon emittance (diffraction limit) reached at such storage rings is $\lambda / 2 \pi$. Using mode decomposition it is shown that, for the parameters of the storage ring considered in this work, the diffraction limit will be reached for soft X-ray energies of $500 \mathrm{eV}$. About ten modes will contribute to the radiation field at $12 \mathrm{keV}$ photon energy and even more modes give a contribution at higher photon energies. Energy spread effects of the electron beam in a low-emittance storage ring were analysed in detail. Simulations were performed at different relative energy spread values from zero to $2 \times 10^{-3}$. A decrease of the degree of coherence with an increase of the relative energy spread value was observed. This analysis shows that, to reach the diffraction limit for high photon energies, electron beam emittance should go down to $1 \mathrm{pm}$ rad and below.

\section{Introduction}

Recently it was realized that, due to a new conceptual approach, i.e. the multi-bend achromat synchrotron storage ring design, the brightness of next-generation $\mathrm{X}$-ray storage rings may be increased by two to three orders of magnitude (Eriksson et al., 2014; Hettel, 2014). This immediately implies that the coherent flux of these storage rings will be higher by two orders of magnitude as well and, by that, will be approaching the so-called diffraction limit. Next-generation synchrotron sources have the potential to make a great contribution to today's major challenges in investigation of multi-functional hybrid materials, electronic transport phenomena and electrochemical processes in charge storage materials under working conditions, as well as materials under extreme conditions of pressure and temperature with highest resolution and sensitivity (Weckert, 2015).

The first storage ring constructed using multi-bend achromat technology was the $3 \mathrm{GeV}$ synchrotron source MAX IV (Lund, Sweden), which recently reached its planned specifications of horizontal emittance of about 200$330 \mathrm{pm}$ rad (depending on insertion device gap settings) (Tavares et al., 2014). The high-energy ESRF $6 \mathrm{GeV}$ storage ring is presently under reconstruction to the EBS ESRF facility (https://www.esrf.eu/home/UsersAndScience/ Accelerators/ebs---extremely-brilliant-source/ebs-parameters. html) that is planned to reach a horizontal emittance of $133 \mathrm{pm}$ rad. The Brazilian SIRIUS $3 \mathrm{GeV}$ project is presently in the commissioning phase with horizontal emittance planned 
in the range 150-250 pm rad (Rodrigues et al., 2018), and other facilities worldwide (APS-U, SPring-8, ALS, Soleil, Diamond, etc.) are in construction or at the planning stage. At DESY in Hamburg, Germany, an upgrade of the high-energy $6 \mathrm{GeV}$ storage ring PETRA III to the PETRA IV facility is also planned (Schroer et al., 2019). The world's lowest emittance of about $10 \mathrm{pm}$ rad for hard X-rays is targeted at this storage ring (Schroer et al., 2018).

Source brilliance and coherence of the future storage rings are the keys for successful synchrotron radiation experiments. The high degree of coherence will allow the focusing of the synchrotron beams efficiently to the nanometre range without spatial filtering of flux (Singer \& Vartanyants, 2014). It will allow an effective application of coherence-based techniques such as coherent diffraction imaging (CDI) potentially reaching sub-nanometre resolution (Schroer \& Falkenberg, 2014). It will also extend photon correlation techniques into the regime of nanoseconds and allow for low dose correlation experiments (Shpyrko, 2014).

In order to reach all these goals, a better understanding of the coherence properties of radiation close to the diffraction limit is necessary. Ultimate storage rings are expected to have a high degree of coherence, which means that traditional methods of X-ray tracing will not be sufficient to predict parameters of X-ray beams at the experimental stations. Also, some intrinsic electron beam parameters such as an unavoidable energy spread of electrons in a storage ring may influence the coherence properties of X-ray beams.

It is interesting to note that even basic parameters of diffraction-limited sources are not well understood nowadays. For example, it is a long-standing debate on what is a correct asymptotic limit for the photon emittance of a diffractionlimited storage ring (Walker, 2019). It is commonly accepted that the diffraction-limited storage ring is the one with its electron beam emittance much lower than the natural emittance of single-electron radiation. For radiation described by the Gaussian functions, the diffraction-limited emittance is very well known from the uncertainty principle and is given by $\lambda / 4 \pi$, where $\lambda$ is the wavelength of radiation (Kim, 1989). At the same time, several authors have demonstrated that the emittance of single-electron undulator radiation is given by $\lambda / 2 \pi$ (Onuki \& Elleaume, 2003; Tanaka \& Kitamura, 2009). The problem is based on the fact that radiation from a single electron cannot be described by a Gaussian function. It is well known that in the far-field region and resonant conditions it is described by a sinc-function (Alferov et al., 1973; Kim, 1986). Further on, we will carefully analyze this question and will give a definitive answer to this long-standing debate. ${ }^{1}$

Another important question is the influence of the energy spread of electrons in a storage ring on the coherence properties of the photon beams. It was first outlined by Tanaka \&

\footnotetext{
${ }^{1}$ When this work was finished the paper written by Walker (Walker, 2019) was published. The thorough analysis presented by the author was based on the synchrotron radiation simulations presented in the book by Onuki \& Elleaume (2003). The author also discussed the question of diffraction limit and came to a similar conclusion as in our work for the so-called projected values of emittance.
}

Kitamura (2009) that energy spread effects may affect the brightness of the X-ray storage rings. In a recent paper by Geloni et al. (2018), energy spread effects on brightness and coherence were carefully analysed for the low-emittance storage rings using an analytical approach. As a result of this analysis, it was shown that the brightness of diffraction-limited sources with small but finite emittance only slightly depends on the energy spread effects; the same is valid for the coherence function centred at the optical axis of the undulator with stronger effects at off-axis values. It was also demonstrated that the values of brightness obtained by this approach substantially differ from the results of Tanaka \& Kitamura (2009). We will discuss in detail the effect of energy spread on the coherence properties of the low-emittance storage rings.

Presently it is a big demand to develope adequate and effective methods that may correctly describe properties of radiation from the ultimate storage rings close to the diffraction limit. Clearly, such a description should be based on the application of the first- and higher-order correlation functions (Mandel \& Wolf, 1995; Vartanyants \& Singer, 2016). Presently several codes have been developed to describe partial coherence radiation from conventional storage rings (see, for example, Chubar \& Elleaume, 1998; Lee \& Zhang, 2007; Shi et al., 2014; Meng et al., 2015). Unfortunately, these codes may be inefficient for simulating properties of diffraction-limited storage rings or require a substantial computer power and time to perform simulations. In this work for the analysis of coherence properties of the low-emittance storage rings, we used the recently developed computer code $X R T$ (Klementiev \& Chernikov, 2014) that supports parallel calculations on GPU and allows efficient simulation of all necessary correlation functions.

In this work, we analysed coherence properties of a highenergy low-emittance (10 pm rad and below) storage ring for different photon energies starting from soft X-rays of $500 \mathrm{eV}$ up to hard X-rays of $50 \mathrm{keV}$. We introduced energy spread effects in our simulations and specifically examined the following values: zero energy spread value, $1 \times 10^{3}$ and $2 \times 10^{3}$. These studies may be of particular interest for the future PETRA IV facility with its record parameters (Schroer et al., 2018).

The paper is organized as follows. In the next section, a short theoretical summary of the first-order correlation functions will be presented. In the same section, the basic principles of undulator radiation and its analytical description will be discussed. In the third and main section of the paper, simulations and results of the analysis of coherence properties of the low-emittance storage ring will be performed by different analysis tools. The paper will be finished with conclusions and outlook.

\section{Theory}

\subsection{Basics of coherence theory}

The measure of the first-order coherence is given by the mutual coherence function (MCF) defined as (Mandel \& Wolf, 1995) 


$$
\Gamma\left(\mathbf{r}_{1}, \mathbf{r}_{2}, t_{1}, t_{2}\right)=\left\langle E^{*}\left(\mathbf{r}_{1}, t_{1}\right) E\left(\mathbf{r}_{2}, t_{2}\right)\right\rangle .
$$

It describes correlations between two values of the electric field, $E\left(\mathbf{r}_{1}, t_{1}\right)$ and $E\left(\mathbf{r}_{2}, t_{2}\right)$, at different points $\mathbf{r}_{1}$ and $\mathbf{r}_{2}$ and times $t_{1}$ and $t_{2}$. The brackets $\langle\ldots\rangle$ denote the ensemble average; for stationary sources the ensemble average coincides with the time average. We next introduce the crossspectral density function (CSD) of a stationary source, which is obtained as the Fourier transform of the MCF (Mandel \& Wolf, 1995),

$$
W\left(\mathbf{r}_{1}, \mathbf{r}_{2}, \omega\right)=\int \Gamma\left(\mathbf{r}_{1}, \mathbf{r}_{2}, \tau\right) \exp (-i \omega \tau) \mathrm{d} \tau,
$$

where $\tau=t_{2}-t_{1}$. The spectral density of the radiation field is obtained when two points $\mathbf{r}_{1}$ and $\mathbf{r}_{2}$ coincide, $\mathbf{r}=\mathbf{r}_{1}=\mathbf{r}_{2}$,

$$
S(\mathbf{r}, \omega)=W(\mathbf{r}, \mathbf{r}, \omega) .
$$

A convenient measure of spatial coherence is the normalized CSD,

$$
\mu\left(\mathbf{r}_{1}, \mathbf{r}_{2}, \omega\right)=\frac{W\left(\mathbf{r}_{1}, \mathbf{r}_{2}, \omega\right)}{\sqrt{S\left(\mathbf{r}_{1}, \omega\right)} \sqrt{S\left(\mathbf{r}_{2}, \omega\right)}},
$$

which is called the spectral degree of coherence (SDC). The values of this function, which are ranging from zero to one and depend on the pinhole separation $\mathbf{r}_{1}$ and $\mathbf{r}_{2}$, are determined in the classical Young's experiment.

Another convenient measure of coherence is the global degree of coherence $\zeta^{\mathrm{DC}}$, which characterizes the coherence properties of the wavefield by a single number and can be introduced as (Geloni et al., 2008; Vartanyants \& Singer, 2010)

$$
\zeta^{\mathrm{DC}}(\omega)=\frac{\int\left|W\left(\mathbf{r}_{1}, \mathbf{r}_{2}, \omega\right)\right|^{2} \mathrm{~d} \mathbf{r}_{1} \mathrm{~d} \mathbf{r}_{2}}{\left[\int S(\mathbf{r}, \omega) \mathrm{d} \mathbf{r}\right]^{2}} .
$$

The values of the parameter $\zeta^{\mathrm{DC}}(\omega)$ lie in the range $0 \leq$ $\zeta^{\mathrm{DC}}(\omega) \leq 1$, where $\zeta^{\mathrm{DC}}(\omega)=1$ and $\zeta^{\mathrm{DC}}(\omega)=0$ correspond to fully coherent and incoherent radiation, respectively.

In the quasi-monochromatic regime, it is possible to approximate the MCF in equation (1) as (Mandel \& Wolf, 1995)

$$
\Gamma\left(\mathbf{r}_{1}, \mathbf{r}_{2}, \tau\right) \simeq J\left(\mathbf{r}_{1}, \mathbf{r}_{2}\right) \exp \left(-i \omega_{0} \tau\right)
$$

provided that $|\tau| \ll 2 \pi / \Delta \omega$, where $\Delta \omega$ is the bandwidth of radiation. Here $J\left(\mathbf{r}_{1}, \mathbf{r}_{2}\right)$ is the mutual optical intensity (MOI) defined as

$$
J\left(\mathbf{r}_{1}, \mathbf{r}_{2}\right) \equiv \Gamma\left(\mathbf{r}_{1}, \mathbf{r}_{2}, 0\right)=\left\langle E^{*}\left(\mathbf{r}_{1}, t\right) E\left(\mathbf{r}_{2}, t\right)\right\rangle .
$$

Taking into account all of the above, we may represent CSD for quasi-monochromatic radiation as

$$
W\left(\mathbf{r}_{1}, \mathbf{r}_{2}, \omega\right)=J\left(\mathbf{r}_{1}, \mathbf{r}_{2}\right) \delta\left(\omega-\omega_{0}\right),
$$

which describes correlations between two complex values of the electric field at different points $\mathbf{r}_{1}$ and $\mathbf{r}_{2}$ at a given frequency.

Finally, for quasi-monochromatic radiation, CSD $W\left(\mathbf{r}_{1}, \mathbf{r}_{2}, \omega\right)$ and MOI $J\left(\mathbf{r}_{1}, \mathbf{r}_{2}\right)$ functions as well as spectral density $S(\mathbf{r}, \omega)$ and intensity $I(\mathbf{r}) \equiv \Gamma(\mathbf{r}, \mathbf{r}, 0)=\left\langle E^{*}(\mathbf{r}, t) E(\mathbf{r}, t)\right\rangle$ functions are equivalent.
2.2. Coherent-mode representation of the cross-spectral density function

It is well known (Mandel \& Wolf, 1995) that, under very general conditions, one can represent the CSD of a partially coherent, statistically stationary field of any state of coherence as a series

$$
W\left(\mathbf{r}_{1}, \mathbf{r}_{2}, \omega\right)=\sum_{j} \beta_{j}(\omega) E_{j}^{*}\left(\mathbf{r}_{1}, \omega\right) E_{j}\left(\mathbf{r}_{2}, \omega\right) .
$$

Here $\beta_{j}(\omega)$ are eigenvalues and independent coherent modes $E_{j}(\mathbf{r}, \omega)$ are eigen-functions of the Fredholm integral equation of the second kind,

$$
\int W\left(\mathbf{r}_{1}, \mathbf{r}_{2}, \omega\right) E_{j}\left(\mathbf{r}_{1}, \omega\right) \mathrm{d} \mathbf{r}_{1}=\beta_{j}(\omega) E_{j}\left(\mathbf{r}_{2}, \omega\right) .
$$

According to equations (3) and(9) the spectral density can be represented as

$$
S(\mathbf{r}, \omega)=\sum_{j} \beta_{j}(\omega)\left|E_{j}(\mathbf{r}, \omega)\right|^{2} .
$$

Substitution of equations (9) and (11) into equation (5) gives, for the global degree of coherence,

$$
\zeta^{\mathrm{DC}}(\omega)=\frac{\sum \beta_{j}^{2}(\omega)}{\left[\sum \beta_{j}(\omega)\right]^{2}} .
$$

One has to remember important characteristics of this coherent mode decomposition: the mode functions $E_{j}(\mathbf{r}, \omega)$ form an orthonormal set, the eigenvalues $\beta_{j}(\omega)$ are real and non-negative, $\beta_{j}(\omega) \geq 0$ and $\beta_{0}(\omega) \geq \beta_{1}(\omega) \geq \ldots$ If there is only one single mode present then radiation is fully coherent. Thus we can define the coherent fraction (CF) of radiation $\zeta^{\mathrm{CF}}(\omega)$ as an occupation or normalized weight of the first mode,

$$
\zeta^{\mathrm{CF}}(\omega)=\frac{\beta_{0}(\omega)}{\sum_{j=0}^{\infty} \beta_{j}(\omega)}
$$

We will consider a quasi-monochromatic case and will omit frequency dependence in the following.

\subsection{Gaussian Schell-model sources}

The Gaussian Schell-model (GSM) is a simplified but often used model (Vartanyants \& Singer, 2010) that represents radiation from a real X-ray source based on the following approximations. The source is modelled as a plane twodimensional source, the source is spatially uniform, i.e. the SDC depends only on the difference $\mathbf{r}_{2}-\mathbf{r}_{1}$, the SDC $\mu\left(\mathbf{r}_{2}-\mathbf{r}_{1}\right)$ and spectral density $S(\mathbf{r})$ are Gaussian functions. In the frame of the GSM cross-spectral density function, spectral density and SDC are defined as (Mandel \& Wolf, 1995)

$$
\begin{gathered}
W\left(\mathbf{r}_{1}, \mathbf{r}_{2}\right)=\mu\left(\mathbf{r}_{2}-\mathbf{r}_{1}\right) \sqrt{S\left(\mathbf{r}_{1}\right)} \sqrt{S\left(\mathbf{r}_{2}\right),} \\
S(\mathbf{r})=S_{0} \exp \left(-\frac{r_{x}^{2}}{2 \sigma_{x}^{2}}-\frac{r_{y}^{2}}{2 \sigma_{y}^{2}}\right),
\end{gathered}
$$




$$
\mu\left(\mathbf{r}_{2}-\mathbf{r}_{1}\right)=\exp \left[-\frac{\left(r_{x 2}-r_{x 1}\right)^{2}}{2 \xi_{x}^{2}}-\frac{\left(r_{y 2}-r_{y 1}\right)^{2}}{2 \xi_{y}^{2}}\right],
$$

where $S_{0}$ is a normalization constant, $\sigma_{x, y}$ is the r.m.s. source size and $\xi_{x, y}$ is the transverse coherence length in the source plane in the $x$ - and $y$-direction, respectively. One of the important features of this model is that the CSD function is separable into two transverse directions,

$$
W\left(\mathbf{r}_{1}, \mathbf{r}_{2}\right)=W\left(r_{x 1}, r_{x 2}\right) W\left(r_{y 1}, r_{y 2}\right) .
$$

The same is valid for the global degree of coherence defined in equation (5),

$$
\zeta^{\mathrm{DC}}=\zeta_{x}^{\mathrm{DC}} \zeta_{y}^{\mathrm{DC}}
$$

where in each transverse direction $i=x, y$ we have (Vartanyants \& Singer, 2010)

$$
\zeta_{i}^{\mathrm{DC}}=\frac{\int\left|W\left(r_{i 1}, r_{i 2}\right)\right|^{2} \mathrm{~d} r_{i 1} \mathrm{~d} r_{i 2}}{\left[\int S\left(r_{i}\right) \mathrm{d} r_{i}\right]^{2}}=\frac{\xi_{i} / \sigma_{i}}{\sqrt{\left(\xi_{i} / \sigma_{i}\right)^{2}+4}} .
$$

Coherent modes in the GSM are described by the HermiteGaussian functions (Gori, 1983; Starikov \& Wolf, 1982; Vartanyants \& Singer, 2010),

$$
\begin{gathered}
\beta_{j} / \beta_{0}=\kappa^{j}, \\
E_{j}\left(r_{i}\right)=\left(\frac{\kappa_{i}}{2 \pi k \sigma^{2} \zeta_{i}}\right)^{1 / 4} \frac{1}{\sqrt{2^{j} j !}} H_{j}\left(\frac{r_{i}}{\sigma \sqrt{2 \zeta_{i}}}\right) \exp \left(-\frac{r_{i}^{2}}{4 \sigma^{2} \zeta_{i}}\right),
\end{gathered}
$$

where the coefficient $\kappa_{i}=\left(1-\zeta_{i}\right) /\left(1+\zeta_{i}\right)$ is introduced, $H_{j}\left(r_{i} / \sigma \sqrt{2 \zeta_{i}}\right)$ are the Hermite polynomials of order $j, k=2 \pi / \lambda$ and $i=x, y$. The zero mode is a Gaussian function and propagation of Hermite-Gaussian modes in the far-field region gives Hermite-Gaussian modes of the same shape. In the frame of GSM, according to equations (13) and (20) the coherent fraction of the radiation for one transverse direction may be determined as

$$
\zeta_{i}^{\mathrm{CF}}=\left[\sum_{j=0}^{\infty} \frac{\beta_{j}(\omega)}{\beta_{0}(\omega)}\right]^{-1}=\left(\sum_{j=0}^{\infty} \kappa^{j}\right)^{-1}=\frac{2 \zeta_{i}}{1+\zeta_{i}} .
$$

\section{Synchrotron radiation from undulator sources}

\subsection{Spectral brightness and phase space distribution}

The source may be completely characterized by its spectral brightness which is defined through the phase space distribution function that is a classical analogue of the Wigner distribution function (Wigner, 1932). According to this definition, the spectral brightness $B\left(\mathbf{r}, \mathbf{k}_{\perp}\right)$ in the paraxial approximation is given by (Mandel \& Wolf, 1995)

$$
\begin{aligned}
B\left(\mathbf{r}, \mathbf{k}_{\perp}\right)= & \left(\frac{k}{2 \pi}\right)^{2} \int W_{0}\left(\mathbf{r}-\frac{1}{2} \Delta \mathbf{r}, \mathbf{r}+\frac{1}{2} \Delta \mathbf{r}\right) \\
& \times \exp \left(i \mathbf{k}_{\perp} \cdot \Delta \mathbf{r}\right) \mathrm{d} \Delta \mathbf{r},
\end{aligned}
$$

where $W_{0}\left(\mathbf{r}-\frac{1}{2} \Delta \mathbf{r}, \mathbf{r}+\frac{1}{2} \Delta \mathbf{r}\right)$ is the CSD function [equations (2) and (8)] defined at the source position, $\mathbf{k}_{\perp}$ is a projection of the momentum vector $\mathbf{k}$ on the transverse plane, and the coordinates $\mathbf{r}$ and $\Delta \mathbf{r}$ are introduced as $\mathbf{r}=\left(\mathbf{r}_{1}+\mathbf{r}_{2}\right) / 2$ and $\Delta \mathbf{r}=\left(\mathbf{r}_{1}-\mathbf{r}_{2}\right)$.

In the synchrotron radiation community, it is conventional to define the distribution function in equation (23) in the phase space through the CSD function $W_{0}(\mathbf{r}, \Delta \mathbf{r})$ of the electric fields at the source position (Kim, 1986, 1989; Geloni et al., 2015),

$$
B(\mathbf{r}, \boldsymbol{\theta})=\frac{c}{(2 \pi)^{4}} \frac{I}{e \hbar} k^{2} \int W_{0}(\mathbf{r}, \Delta \mathbf{r}) \exp (-i k \boldsymbol{\theta} \cdot \Delta \mathbf{r}) \mathrm{d} \Delta \mathbf{r},
$$

where $\boldsymbol{\theta}$ is the angle between the optical axis and observation direction, $I$ is the electron beam current, $e$ is an electron charge, and $h=h / 2 \pi$ where $h$ is Planck's constant. This distribution taken at its maximum value, which typically for undulator radiation coincides with the optical axis for odd harmonics, is defined as the spectral brightness of the synchrotron source.

Approximating the far-field distribution of the electric field of undulator radiation in resonant conditions from a single electron by a Gaussian laser mode the following well known approximation for the brightness of undulator radiation was obtained (Kim, 1986, 1989),

$$
B_{0}=B(0,0)=\frac{1}{4 \pi^{2}} \frac{F}{\Sigma_{\mathrm{ph} x} \Sigma_{\mathrm{ph} x}^{\prime} \Sigma_{\mathrm{ph} y} \Sigma_{\mathrm{ph} y}^{\prime}},
$$

where $F$ is the spectral photon flux into the central core. In equation (25), the total photon source size $\Sigma_{\mathrm{ph}}$ and divergence $\Sigma_{\mathrm{ph}}^{\prime}$ are defined as

$$
\Sigma_{\mathrm{ph}} \simeq\left(\sigma_{\mathrm{e}}^{2}+\sigma_{\mathrm{r}}^{2}\right)^{1 / 2}, \quad \Sigma_{\mathrm{ph}}^{\prime} \simeq\left(\sigma_{\mathrm{e}}^{\prime 2}+\sigma_{\mathrm{r}}^{\prime 2}\right)^{1 / 2},
$$

where $\sigma_{\mathrm{e}}=\sqrt{\varepsilon_{\mathrm{e}} \beta_{\mathrm{e}}}$ and $\sigma_{\mathrm{e}}^{\prime}=\sqrt{\varepsilon_{\mathrm{e}} / \beta_{\mathrm{e}}}$ are the electron beam spatial size and angular divergence ${ }^{2}, \varepsilon_{\mathrm{e}}=\sigma_{\mathrm{e}} \sigma_{\mathrm{e}}^{\prime}$ is the emittance of an electron beam at its waist, and $\beta_{\mathrm{e}}$ is the value of the betatron function in the centre of the undulator. Intrinsic characteristics of single-electron radiation in equation (26) were introduced by Kim (1989),

$$
\sigma_{\mathrm{r}}=\frac{\sqrt{2 \lambda_{n} L_{\mathrm{u}}}}{4 \pi}, \quad \sigma_{\mathrm{r}}^{\prime}=\sqrt{\frac{\lambda_{n}}{2 L_{\mathrm{u}}}},
$$

where $\lambda_{n}$ and $L_{\mathrm{u}}$ are the $n$th harmonic radiation wavelength and the undulator length. The total photon emittance of the undulator source is then introduced as

$$
\varepsilon_{\mathrm{ph} x, y}=\Sigma_{\mathrm{ph} x, y} \Sigma_{\mathrm{ph} x, y}^{\prime}
$$

\footnotetext{
2 This expression for the electron beam spatial size and angular divergence is valid when electron beam dispersion is neglected in a straight section.
} 
By that approach, the photon emittance $\varepsilon_{\mathrm{r}}$ or photon phase space of a single electron in a Gaussian approximation is given by the value (Kim, 1989)

$$
\varepsilon_{\mathrm{r}}=\sigma_{\mathrm{r}} \sigma_{\mathrm{r}}^{\prime}=\lambda / 4 \pi
$$

The diffraction-limited undulator source may be defined as the one with the electron beam parameters satisfying the following inequalities: $\sigma_{\mathrm{e}} \ll \sigma_{\mathrm{r}}$ and $\sigma_{\mathrm{e}}^{\prime} \ll \sigma_{r}^{\prime}$. According to these definitions for the diffraction-limited source, the natural electron beam emittance has to be much smaller than the natural emittance of single-electron radiation, $\varepsilon_{\mathrm{e}} \ll \varepsilon_{\mathrm{r}} \equiv \lambda / 4 \pi$.

\subsection{Energy spread effects}

The energy spread of the electron beam is an energy distribution of electrons in the bunch, which for synchrotron radiation obeys Gaussian statistics. As noted in several publications (Onuki \& Elleaume, 2003; Tanaka \& Kitamura, 2009), energy spread effects may influence the properties of the synchrotron radiation source. By approximating the angular and spatial profile of the flux density by Gaussian functions for each transverse direction, the following expressions for the beam size and divergence were obtained (Tanaka \& Kitamura, 2009),

$$
\begin{aligned}
& \Sigma_{\mathrm{ph}} \simeq\left[\varepsilon \beta+\sigma_{\mathrm{r}}^{2} 4 N(v / 4)^{2 / 3}\right]^{1 / 2}, \\
& \Sigma_{\mathrm{ph}}^{\prime} \simeq\left[(\varepsilon / \beta)+\sigma_{\mathrm{r}}^{\prime 2} N(v)\right]^{1 / 2},
\end{aligned}
$$

where $\sigma_{\mathrm{r}}$ and $\sigma_{\mathrm{r}}^{\prime}$ are intrinsic characteristics of single-electron radiation, defined in equations (27). A normalization factor $N(v)$ for the energy spread of the storage ring in equations (30) is defined as

$$
N(v)=\frac{8 \pi^{2} v^{2}}{(2 \pi)^{3 / 2} v \operatorname{erf}\left[\left(8 \pi^{2}\right)^{1 / 2} v\right]+\exp \left(-8 \pi^{2} v\right)-1},
$$

with $v=\delta_{\gamma} / \delta_{n}$ being the ratio between the relative energy spread value $\delta_{\gamma}=\Delta E / E$ and the relative bandwidth $\delta_{n}=$ $1 / n N_{\mathrm{u}}$ of the $n$th harmonic of an undulator with $N_{\mathrm{u}}$ periods. The normalization function $N(v)$ for zero relative energy spread is equal to one, $N(0)=1$. Note, that the source size for natural single-electron radiation in equation (30) is effectively two times larger than defined in equation (27). This difference originates from a non-Gaussian angular profile of far-field radiation from a single electron that is taken into account in equation (30). This also leads to a relaxed condition for the diffraction-limited source: $\varepsilon_{\mathrm{e}} \ll \varepsilon_{\mathrm{r}}=2 \sigma_{\mathrm{r}} \sigma_{\mathrm{r}}^{\prime} \equiv \lambda / 2 \pi$. We will see in the following, by performing simulations, which of two conditions is satisfied in the limit of small electron beam emittance for the diffraction-limited undulator source.

In a recent paper by Geloni et al. (2018), energy spread effects on brightness and coherence were carefully analysed using directly equation (24) with an account of energy spread effects. For the far-field distribution of the electric field emitted by an electron with the energy deviating from the resonant photon energy of the undulator radiation harmonic, the following expression was used in equation (24) (Geloni et al., 2018),

$$
\begin{aligned}
E(\boldsymbol{\theta})= & -\frac{K \omega e L_{\mathrm{u}}}{2 c^{2} z \gamma} A_{j j} \exp \left[i \frac{\omega}{c}\left(\frac{z \boldsymbol{\theta}^{2}}{2}-\boldsymbol{\theta} \cdot \boldsymbol{l}\right)\right] \\
& \times \operatorname{sinc}\left[\frac{2 \pi N_{\mathrm{u}}\left(\gamma-\gamma_{1}\right)}{\gamma_{1}}+\frac{\omega L_{\mathrm{u}}|\boldsymbol{\theta}-\boldsymbol{\eta}|^{2}}{4 c}\right],
\end{aligned}
$$

where $\gamma$ and $\gamma_{1}$ are the Lorentz factors at a given and resonant frequency electron energy, $z$ is the distance from the centre of the undulator source, $K$ is the maximum undulator deflection parameter, and the coupling parameter $A_{j j}$ is defined as

$$
A_{j j}=J_{0}\left(\frac{K^{2}}{4+2 K^{2}}\right)-J_{1}\left(\frac{K^{2}}{4+2 K^{2}}\right),
$$

where $J_{n}$ is the $n$ th-order Bessel function of the first kind. In expression (32), energy offset $\Delta \gamma=\gamma-\gamma_{1}$, different entering angles $\boldsymbol{\eta}$ and various axis offset $\boldsymbol{l}$ of the electron in the undulator are explicitly taken into account.

In our further analysis, we will need the amplitude of the field at the source position. It is determined directly from expression (32) by applying the propagator in free space in paraxial approximation and is given by the following expression (Geloni et al., 2015),

$$
E_{0}(\mathbf{r})=\frac{i z \omega}{2 \pi c} \int E(\boldsymbol{\theta}) \exp \left(-\frac{i \theta^{2} z \omega}{2 c}\right) \exp \left(\frac{i \omega \boldsymbol{r} \cdot \boldsymbol{\theta}}{c}\right) \mathrm{d} \boldsymbol{\theta} .
$$

To obtain the source size and divergence for non-Gaussian field distributions we determined them by calculating the second moments or variances of the intensity distribution of the corresponding variables in each direction, respectively (Onuki \& Elleaume, 2003),

$$
\begin{aligned}
\Sigma_{\mathrm{ph}_{x}}^{2} & =\frac{\iint x^{2}\left|E_{0}(x, y)\right|^{2} \mathrm{~d} x \mathrm{~d} y}{\iint\left|E_{0}(x, y)\right|^{2} \mathrm{~d} x \mathrm{~d} y}, \\
\Sigma_{\mathrm{ph} x}^{\prime 2} & =\frac{\iint \theta_{x}^{2}\left|E\left(\theta_{x}, \theta_{y}\right)\right|^{2} \mathrm{~d} \theta_{x} \mathrm{~d} \theta_{y}}{\iint\left|E\left(\theta_{x}, \theta_{y}\right)\right|^{2} \mathrm{~d} \theta_{x} \mathrm{~d} \theta_{y}} .
\end{aligned}
$$

In addition to energy spread effects, the source size and source divergence are influenced by undulator detuning - a shift of the undulator harmonic wavelength $\lambda_{n}$ relative to the selected radiation wavelength $\lambda$ (Coisson, 1988). Detuning of the radiation wavelength from the resonant undulator harmonic value towards lower energies is very often used in practice to gain higher flux than at the resonant photon energy (Onuki \& Elleaume, 2003). Depending on its value, detuning may have a stronger effect (a bigger linear and angular size variation) than energy spread but is out of the scope of the present paper. In this work, the radiation field was calculated in the monochromatic regime at a fixed resonance frequency. As such, no averaging over the frequency values was performed here.

\subsection{Coherent fraction}

It is well established in the synchrotron radiation community that the coherent flux of a zero-emittance beam is given by $F_{\text {coh }}=(\lambda / 2)^{2} B_{0}$ (see, for example, Kim, 1986; Geloni et al., 2015). Importantly, this is an exact result that does not depend 
on the Gaussian approximation of single-electron radiation. Next, if we define the flux of the synchrotron source with certain values of emittance through brightness as in equation (25), we obtain

$$
F=4 \pi^{2} \varepsilon_{\mathrm{ph}}^{x} \varepsilon_{\mathrm{ph}}^{y} B_{0} .
$$

Strictly speaking, this expression is valid only for a Gaussian approximation of the electron-beam source parameters and natural single electron radiation with emittance values $\varepsilon_{\mathrm{ph} x, y}$ defined in equation (28) (see, for discussion, Geloni et al., 2018). We will introduce the coherent fraction as

$$
\zeta^{\mathrm{CF}}=\frac{F_{\mathrm{coh}}}{F}=\left(\frac{\lambda}{4 \pi}\right)^{2} \frac{1}{\varepsilon_{\mathrm{ph}}^{x} \varepsilon_{\mathrm{ph}}^{y}}=\frac{\varepsilon_{\mathrm{r}}^{2}}{\varepsilon_{\mathrm{ph}}^{x} \varepsilon_{\mathrm{ph}}^{y}},
$$

where $\varepsilon_{\mathrm{r}}$ is the conventional emittance of single-electron radiation defined in equation (29). We will see in the following how expression (36) will be modified due to the non-Gaussian behaviour of single-electron radiation in the far-field region.

\section{Results and discussion}

As an example of the diffraction-limited undulator source, we considered a high-energy storage ring operating at $6 \mathrm{GeV}$ (for parameters of the source see Tables 1 and 2). For the electron emittance as a basic value, we considered $10 \mathrm{pm}$ rad both in the vertical and horizontal direction by considering rather a round beam shape. We also analysed a broader range of electron emittance values from $1 \mathrm{pm}$ rad to $300 \mathrm{pm} \mathrm{rad}$ to have a better understanding of the photon properties of the source. Simulations were performed either in the far-field region at the distance of $30 \mathrm{~m}$ from the source or directly at the source position that was considered in the middle of the undulator. Four energy values, $500 \mathrm{eV}, 12 \mathrm{keV}, 24 \mathrm{keV}$ and $50 \mathrm{keV}$, were considered in this work. Comparing the natural emittance of single-electron radiation at these energies with the designed emittance of the source (see Table 2) we see that at the energy of $12 \mathrm{keV}$ emittance values are comparable, at $500 \mathrm{eV}, \varepsilon_{\mathrm{e}} \ll \varepsilon_{\mathrm{r}}$, and at $24 \mathrm{keV}$ as well as at $50 \mathrm{keV}, \varepsilon_{\mathrm{e}}>\varepsilon_{\mathrm{r}}$. From that, we may expect to reach the diffraction limit at $500 \mathrm{eV}$ and have parameters of radiation close to the diffraction-limited source at $12 \mathrm{keV}$. We also would expect that, at higher energies of $24 \mathrm{keV}$ and $50 \mathrm{keV}$, radiation will be highly coherent but not diffraction-limited. All simulations were performed with the XRT software (Klementiev \& Chernikov, 2014) (for details of the $X R T$ software see the supporting information) and compared with the results of analytical calculations.

\subsection{Photon emittance}

Results of simulations performed by the $X R T$ software of the photon emittance as a function of the electron beam emittance values from $1 \mathrm{pm}$ rad to $300 \mathrm{pm}$ rad for different relative energy spread values are presented in Fig. 1. X-ray radiation of the electron beam was simulated for the synchrotron storage ring with the parameters presented in Tables 1 and 2 (for details see the supporting information).
Table 1

Basic simulation parameters of the accelerator and undulator source for all photon energies.

\begin{tabular}{ll}
\hline Electron energy & $6.0 \mathrm{GeV}$ \\
Beam current & $100 \mathrm{~mA}$ \\
Horizontal and vertical electron beam emittance & $10 \mathrm{pm} \mathrm{rad}$ \\
Horizontal and vertical betatron functions, $\beta_{x}, \beta_{y}$ & $2.0 \mathrm{~m}$ \\
Relative energy spread values & $0 ; 1 \times 10^{-3} ; 2 \times 10^{-3}$ \\
Undulator length & $5 \mathrm{~m}$ \\
\hline
\end{tabular}

Table 2

Phase space parameters (in Gaussian approximation) of an X-ray source at different photon energies for $10 \mathrm{pm}$ rad electron beam emittance and

\begin{tabular}{|c|c|c|c|c|}
\hline Photon energy (keV) & 0.5 & 12 & 24 & 50 \\
\hline Number of undulator periods, $N_{\mathrm{u}}$ & 72 & 170 & 170 & 170 \\
\hline Number of harmonics, $n$ & $1 \mathrm{st}$ & $3 r d$ & $3 \mathrm{rd}$ & 5 th \\
\hline $\begin{array}{l}\text { Single electron radiation size, } \sigma_{\mathrm{r}}(\mu \mathrm{m}) \\
\quad \text { [equation }(27) \text { ] }\end{array}$ & 12.5 & 2.55 & 1.79 & 1.25 \\
\hline $\begin{array}{l}\text { Single electron radiation divergence, } \\
\left.\sigma_{\mathrm{r}}^{\prime}(\mu \mathrm{rad}) \text { [equation }(27)\right]\end{array}$ & 15.7 & 3.2 & 2.3 & 1.5 \\
\hline $\begin{array}{l}\text { Single electron radiation emittance, } \\
\varepsilon_{\mathrm{r}}=\lambda / 4 \pi(\mathrm{pm} \mathrm{rad})\end{array}$ & 197 & 8.2 & 4.1 & 1.9 \\
\hline Electron beam size, $\sigma_{\mathrm{e}}(\mu \mathrm{m})$ & 4.47 & 4.47 & 4.47 & 4.47 \\
\hline Electron beam divergence, $\sigma_{\mathrm{e}}^{\prime}(\mu \mathrm{rad})$ & 2.24 & 2.24 & 2.24 & 2.24 \\
\hline $\begin{array}{l}\text { Total photon emittance, } \varepsilon_{\mathrm{ph}}(\mathrm{pm} \mathrm{rad}) \\
\quad \text { equation (26)] }\end{array}$ & 211 & 20 & 15.5 & 12.5 \\
\hline
\end{tabular}
zero energy spread value.
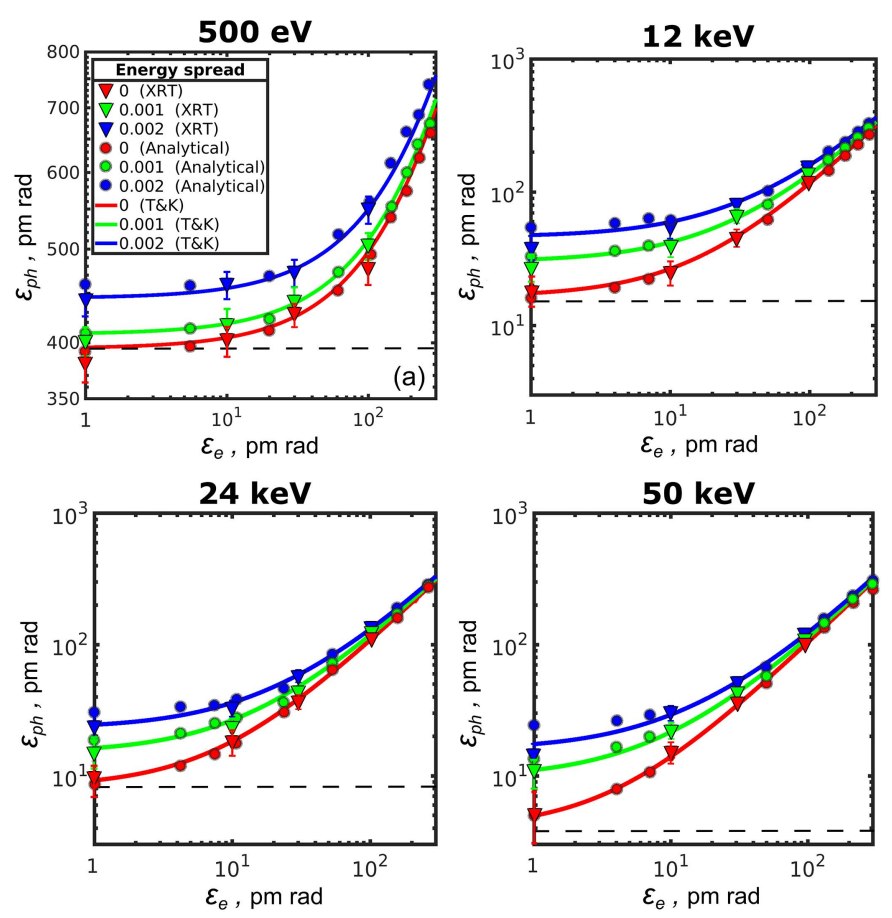

Figure 1

Photon emittance $\varepsilon_{\text {ph }}$ as a function of the electron beam emittance $\varepsilon_{\mathrm{e}}$ for the different values of the photon energy and energy spread in one transverse direction. Triangles are $X R T$ simulations, circles are analytical calculations and lines are the values obtained from the Tanaka \& Kitamura (2009) (T\&K) approach [equations (30) and (31)]. Red, green and blue colour correspond to $0,1 \times 10^{-3}$ and $2 \times 10^{-3}$ relative energy spread values, respectively. Note the different scale for the $500 \mathrm{eV}$ emittance value. The dashed horizontal line corresponds to the value of the photon emittance of $\lambda / 2 \pi$. 
Table 3

Photon emittance values determined at different energies and zero electron energy spread using $X R T$ simulations, an analytical approach and equations (30)-(31).

Results are presented for the $10 \mathrm{pm}$ rad and $1 \mathrm{pm}$ rad electron emittance values.

\begin{tabular}{|c|c|c|c|c|}
\hline Photon energy (keV) & 0.5 & 12 & 24 & 50 \\
\hline$\varepsilon_{\mathrm{coh}}=\lambda / 2 \pi(\mathrm{pm} \mathrm{rad})$ & 395 & 16.4 & 8.2 & 3.9 \\
\hline \multicolumn{5}{|l|}{$10 \mathrm{pm}$ rad natural electron emittance } \\
\hline$\varepsilon_{\mathrm{ph}}^{\mathrm{XRT}}(\mathrm{pm} \mathrm{rad})$ & 398 & 24.6 & 18.5 & 15.2 \\
\hline$\varepsilon_{\mathrm{ph}}^{\text {Analyt. }}(\mathrm{pm} \mathrm{rad})$ & 402 & 24.9 & 17.7 & 13.8 \\
\hline$\varepsilon_{\mathrm{ph}}^{\mathrm{T \& K}}(\mathrm{pm} \mathrm{rad})$ & 403 & 26.5 & 18.3 & 13.8 \\
\hline Source size $(\mathrm{FWHM}), \Sigma_{\mathrm{ph}}^{\text {Analyt. }}(\mu \mathrm{m})$ & 59 & 15.9 & 13.5 & 12 \\
\hline Source divergence $(\mathrm{FWHM}), \Sigma_{\mathrm{ph}}^{\prime \text { Analyt }}(\mu \mathrm{m})$ & 37 & 9.3 & 7.5 & 6.5 \\
\hline \multicolumn{5}{|l|}{$1 \mathrm{pm} \mathrm{rad}$ natural electron emittance } \\
\hline$\varepsilon_{\mathrm{ph}}^{\mathrm{XRT}}(\mathrm{pm} \mathrm{rad})$ & 380 & 20 & 10 & 5 \\
\hline$\varepsilon_{\mathrm{ph}}^{\text {Analytical }}(\mathrm{pm} \mathrm{rad})$ & 391 & 16.3 & 8.9 & 5 \\
\hline$\varepsilon_{\mathrm{ph}}^{\mathrm{T} \& \mathrm{~K}}(\mathrm{pm} \mathrm{rad})$ & 396 & 17.5 & 9.3 & 5 \\
\hline Source size $(\mathrm{FWHM}), \Sigma_{\mathrm{ph}}^{\text {Analyt. }}(\mu \mathrm{m})$ & 58.6 & 12.4 & 9.1 & 6.7 \\
\hline Source divergence $(\mathrm{FWHM}), \Sigma_{\mathrm{ph}}^{\prime \text { Analyt. }}(\mu \mathrm{m})$ & 37.5 & 7.8 & 5.7 & 4.1 \\
\hline
\end{tabular}

The photon emittance (triangles in Fig. 1) was calculated as a product of the source size and divergence [see equation (28)] which were obtained as the variance values of the intensity distributions of the corresponding variables at the source position or in the far-field region according to equations (34).

As a result of these simulations, we can see that the lower the electron emittance $\varepsilon_{\mathrm{e}}$, the lower the photon emittance $\varepsilon_{\mathrm{ph}}$. Importantly, we can observe that at $500 \mathrm{eV}$ and $12 \mathrm{keV}$ photon energies and at $10 \mathrm{pm}$ rad electron beam emittance the photon emittance reaches its asymptotic value (note also the different scale for $500 \mathrm{eV}$ photon energy). This is a clear indication that at these energies the synchrotron source may be considered as diffraction limited. We also observe that, at larger energy spread values and the same electron emittance, photon emittance is also increasing. However, the energy-spread-induced difference does not exceed $12 \%$ at $500 \mathrm{eV} / 10 \mathrm{pm}$ rad, while at higher energies this difference goes up to $50 \%$. This is due to the fact that at low photon energies the properties of the beam (source size and divergence) are comparably large, and their small changes caused by the energy spread effect are not noticeable. In contrast, the energy spread effects are revealed strongly at high energies due to smaller parameters of the radiation at these energies.

We compared these results with the analytical ones obtained using equations (32)-(34) (circles in Fig. 1) (see for details the supporting information). We see that the results of the analytical approach and simulations performed with the $X R T$ software correspond to each other very well (both for different electron emittance values and for different energy spread values) within the margins of the error bars. Both approaches were also compared with the calculations made according to the approach of Tanaka \& Kitamura (2009), equations (30)-(31) (shown by lines in Fig. 1). We observe that all three approaches give similar results for the three values of energy spread that were considered here.

As can be clearly seen in Fig. 1 (see also Table 3), the lowest value of the photon emittance for zero energy spread in our simulations is asymptotically reaching the value of $\lambda / 2 \pi$, when electron emittance values are becoming sufficiently small. This is a strong indication that for low-emittance storage rings $\mathrm{X}$-ray radiation cannot be approximated as Gaussian, because in this case the lowest photon emittance should reach the value of $\lambda / 4 \pi$ as suggested by Kim (1989). At the same time, these results are in concordance with the other results (see, for example, Onuki \& Elleaume, 2003; Tanaka \& Kitamura, 2009), where non-Gaussian behaviour of synchrotron radiation of a single electron was analysed.

Now we would like to determine a coherent fraction of radiation at different electron emittance values. If we use the conventional expression (36) with the emittance values $\varepsilon_{\mathrm{ph}}$ shown in Fig. 1 our coherent fraction values would reach an asymptotic value of 0.5 and would never reach 1 . We slightly redefined our previous expression to the new one (see also Onuki \& Elleaume, 2003),

$$
\zeta^{\mathrm{CF}}=\frac{F_{\mathrm{coh}}}{F}=\frac{\varepsilon_{\mathrm{coh}}^{2}}{\varepsilon_{\mathrm{ph}}^{x} \varepsilon_{\mathrm{ph}}^{y}},
$$

where $\varepsilon_{\mathrm{coh}}=\lambda / 2 \pi$ and $\varepsilon_{\mathrm{ph}}$ are photon emittance values obtained through different simulations. We would like also to note here that definitions of the coherent fraction through equations (13) and (37) are, in fact, equivalent. Indeed, the total flux of radiation $F$ may be represented through the spectral density integrated over the solid angle (see Mandel \& Wolf, 1995). The spectral density may be decomposed into a sum of modes, which are orthonormal. Performing angular integration in this expression will lead to a sum of mode eigenvalues. Following the same arguments, we may represent the coherent flux $F_{\text {coh }}$ as a weight of the zero mode.

The results of our simulations in one transverse direction are presented in Fig. 2. First, we determined the coherent fraction from $X R T$ simulations (triangles) by using expression (37) and the results of emittance simulations shown in Fig. 1. Then we compared the results of $X R T$ simulations with the analytical calculation. Expressions (32) and (33) were used to calculate the wavefield amplitudes in the far-field region and at the source position. Equations (34) were applied to determine the source parameters. Using the same expression (37) we obtained analytical values (circles) of the coherent fraction shown in Fig. 2. Finally, we used in the definition of the coherent fraction [equation (37)] the values provided by equations (30) and (31) (Tanaka \& Kitamura, 2009) (shown by lines).

We see that all three results excellently agree with each other and show the same trend that was just discussed for emittance. It is important to note that to obtain this result we have to use expression (37) instead of the commonly used equation (36). Next, we will turn to a more general definition of coherent fraction through coherent mode decomposition. 

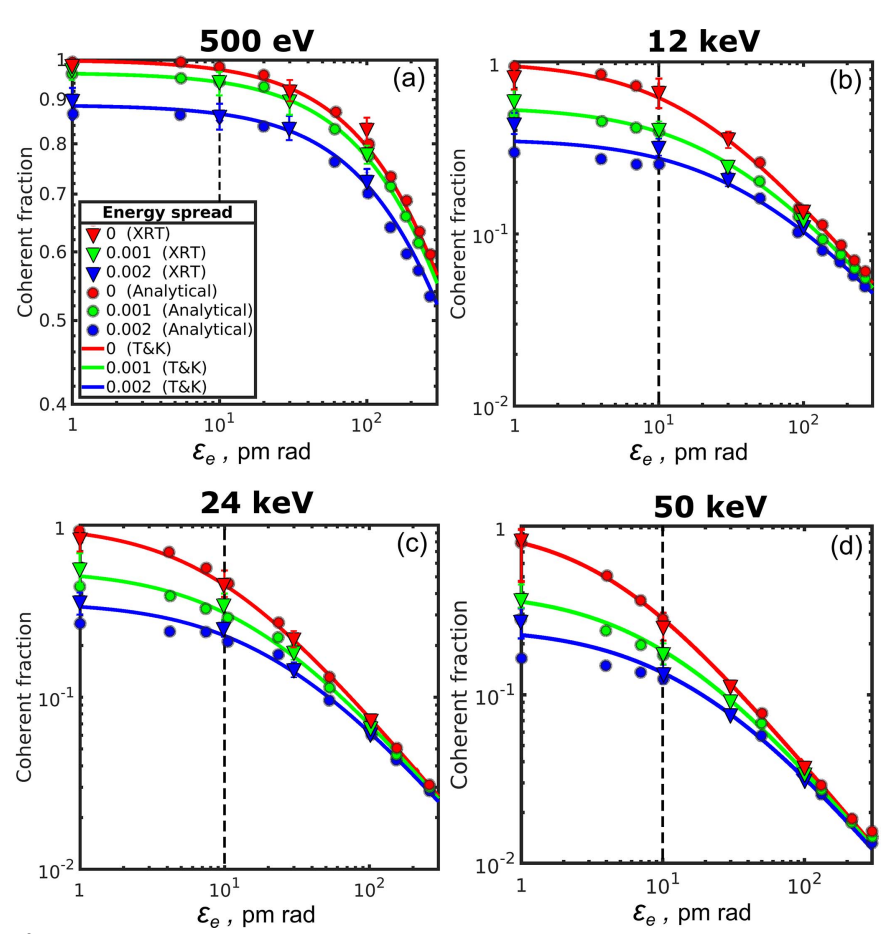

Figure 2

Coherent fraction of radiation $\zeta^{\mathrm{CF}}$ as a function of the electron beam emittance $\varepsilon_{\mathrm{e}}$ for the different values of the photon energy and energy spread in one transverse direction calculated according to equation (37). Triangles are $X R T$ simulations, circles are analytical calculations, and lines are the values obtained by the Tanaka \& Kitamura (2009) (T\&K) approach [equations (30)-(31)]. Red, green and blue colour correspond to $0,1 \times 10^{-3}$ and $2 \times 10^{-3}$ relative energy spread values, respectively. Note the different scale for $500 \mathrm{eV}$ coherent fraction value. Dashed vertical lines correspond to the value of the electron emittance of $10 \mathrm{pm}$ rad.

\subsection{Representation of the cross-spectral density by the coherent mode decomposition}

As discussed in the Theory section, the four-dimensional CSD function may be decomposed to a sum of two-dimensional coherent modes as given in equation (9). The XRT software is providing an opportunity to directly analyze these modes (see the supporting information for details).

We performed mode decomposition of the CSD according to equations (9)-(11) using the XRT software. We performed our analysis for the studied case of $10 \mathrm{pm}$ rad electron beam emittance. The mode decomposition was used to determine the shape and contribution of each mode at different photon energies and at different values of relative energy spread. The first four modes and their normalized weights for $500 \mathrm{eV}$ and $12 \mathrm{keV}$ photon energy are shown in Fig. 3 (results of simulations for $24 \mathrm{keV}$ and $50 \mathrm{keV}$ are given in Fig. S2 of the supporting information). An orthogonal set of modes determined by our simulations, for the whole range of energy spread values from 0 to $2 \times 10^{-3}$, represents a mixture of Laguerre-Gaussian-like and Hermite-Gaussian-like modes (Siegman, 1986). These two different sets of modes have different symmetry: Hermite-Gaussian modes may be represented as a product of two separable amplitude functions in the transverse plane and, contrary to that, Laguerre-Gaussian modes possess cylindrical symmetry and do not allow factorization in two orthogonal directions. It can also be noticed that the contribution of Laguerre-Gaussian modes is increased for both energies with the increase of the energy spread values. It is interesting to note here that the contribution of LaguerreGaussian modes is coming solely from the energy spread effect. It can be shown that if all electron offset parameters (entering angle $\boldsymbol{\eta}$ and axis offset $\boldsymbol{l}$ ) are put to zero then all modes of radiation field may be represented by LaguerreGaussian modes. This all means that, in general, for the diffraction-limited source, it is not possible to factorize the CSD function in two orthogonal transverse directions and define the degree of coherence as a product of its values in each direction [see equation (18)].

We also estimated the number of modes that contribute dominantly to the CSD [equation (9) and their spectral density, equation (11)]. We evaluated the weights of different modes normalized to zero mode $\beta_{j} / \beta_{0}$ and introduced a
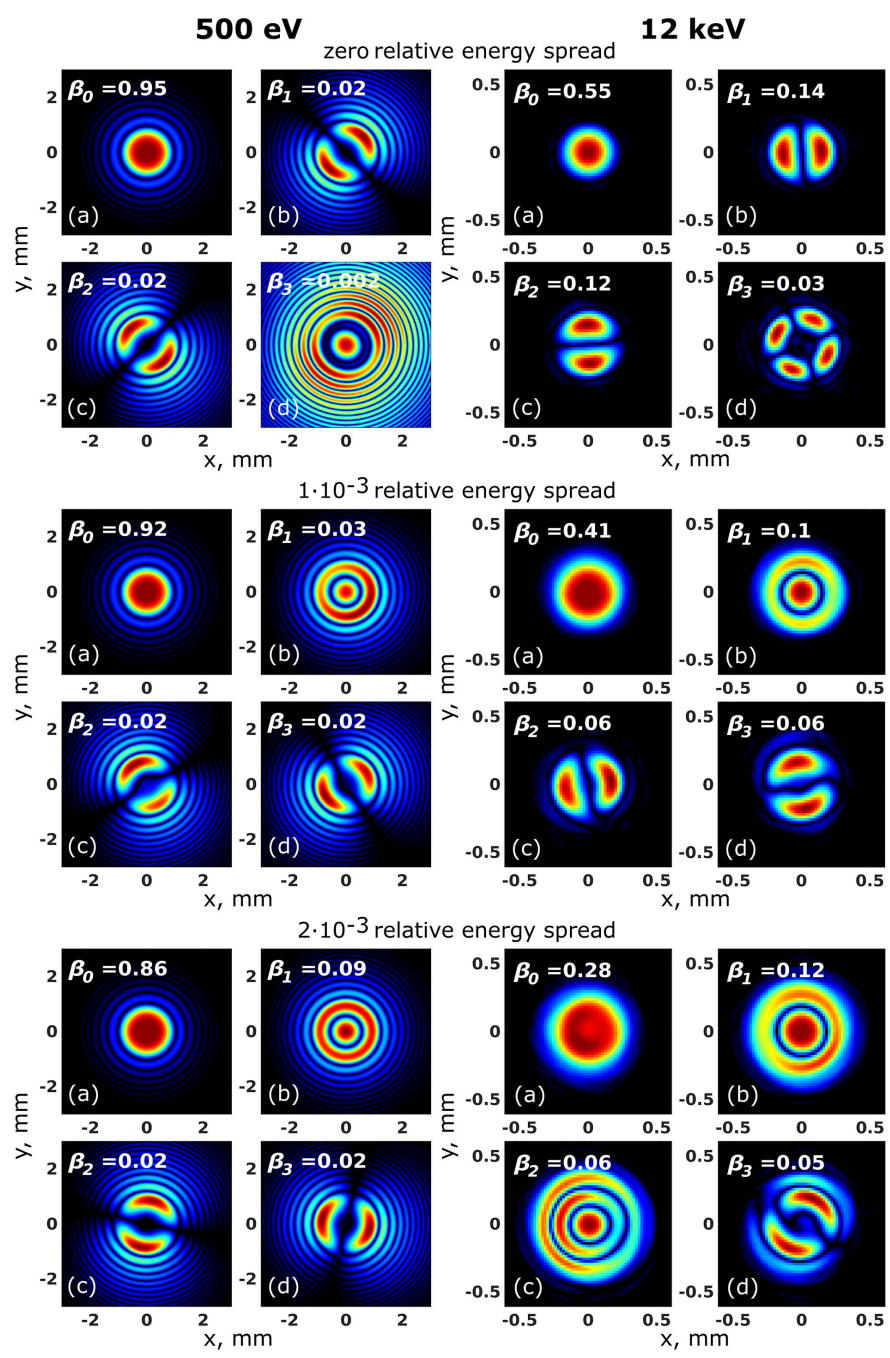

Figure 3

First four modes and their normalized weights $\beta_{j}$ obtained from the coherent mode decomposition of the CSD at $500 \mathrm{eV}$ (left column) and $12 \mathrm{keV}$ (right column) photon energy for three different relative energy spread values obtained by $X R T$ simulations. 
threshold value of $1 \%$. The values of these weights are presented in Fig. 4 as a function of the mode number for all photon energies and energy spread values considered in this work. As can be seen from this figure for $500 \mathrm{eV}$ photon energy and zero energy spread only three modes contribute significantly and this value is increased only to four modes as energy spread values are increased. Already for $12 \mathrm{keV}$ photon energy, the number of modes with a contribution higher than $1 \%$ is about ten. This rises with the increased energy spread values reaching 42 modes at $2 \times 10^{-3}$ relative energy spread value. The number of modes that contribute dominantly to the CSD and spectral density with a threshold of $1 \%$ is 19 for $24 \mathrm{keV}$ and 35 for $50 \mathrm{keV}$ in the zero relative energy spread case. With the increase of energy spread to the value of $2 \times 10^{-3}$ the number of modes contributing dominantly to the CSD is also increasing to 54 at $24 \mathrm{keV}$ and to 90 at $50 \mathrm{keV}$, which is significantly larger than in the previous case of lower photon energies.

As soon as all mode weights were determined by the $X R T$ software, we also determined the global degree of coherence for the considered photon energies and energy spread values according to equation (12) (see Table 4). The values of the global degree of coherence vary from $90 \%$ to $11 \%$ for photon energies from $500 \mathrm{eV}$ to $50 \mathrm{keV}$, respectively. They drop down significantly with the increase of energy spread value for the same range of photon energies.
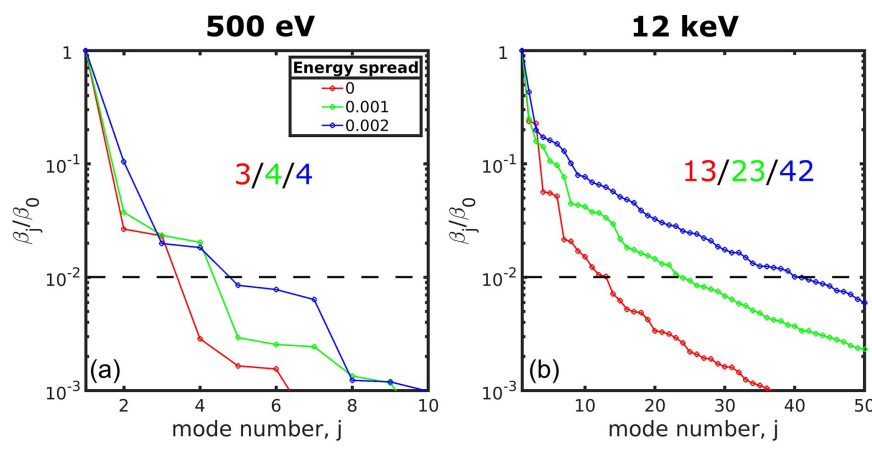

$24 \mathrm{keV}$
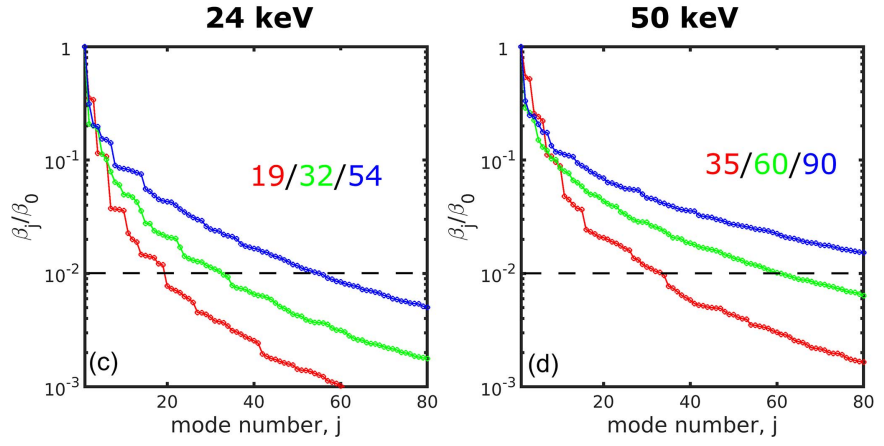

Figure 4

Weights of different modes normalized to the weight of a zero-mode $\beta_{j} / \beta_{0}$ as a function of the mode number $j$ for the different values of the photon energy and energy spread. The horizontal dashed lines correspond to the value of $1 \%$. Points are connected by lines for better visibility. Red, green and blue colour correspond to $0,1 \times 10^{-3}$ and $2 \times$ $10^{-3}$ relative energy spread values, respectively. The number of modes exceeding $1 \%$ threshold is given in each panel.
Table 4

Global degree of coherence [equation (12)] and the coherent fraction [equation (13)] determined from coherent mode decomposition using $X R T$ software and the analytical approach [equations (32) and (33)].

All simulations were performed at the electron beam emittance value of $10 \mathrm{pm}$ rad for different photon energies and energy spread values.

\begin{tabular}{lllll}
\hline Photon energy $(\mathrm{keV})$ & 0.5 & 12 & 24 & 50 \\
\hline $\begin{array}{l}\text { Zero relative energy spread } \\
\quad \text { Global degree of coherence, } \zeta_{\mathrm{XRT}}^{\mathrm{DC}}\end{array}$ & 0.90 & 0.34 & 0.20 & 0.11 \\
$\quad$ Coherent fraction, $\zeta_{\mathrm{XRT}}^{\mathrm{CF}}$ & 0.95 & 0.55 & 0.40 & 0.26 \\
$\quad$ Coherent fraction, $\zeta_{\text {Analyt. }}^{\mathrm{CF}}$ & 0.95 & 0.56 & 0.41 & 0.26 \\
& & & & \\
& & & & \\
$\quad \times 10^{-3}$ relative energy spread & 0.84 & 0.20 & 0.13 & 0.06 \\
$\quad$ Global degree of coherence, $\zeta_{\mathrm{XRT}}^{\mathrm{DC}}$ & 0.91 & 0.41 & 0.35 & 0.22 \\
$\quad$ Coherent fraction, $\zeta_{\mathrm{XRT}}^{\mathrm{CF}}$ & 0.93 & 0.37 & 0.3 & 0.17 \\
$\quad$ Coherent fraction, $\zeta_{\text {Analyt. }}^{\mathrm{CF}}$ & & & & \\
$\quad$ & & & & \\
$2 \times 10^{-3}$ relative energy spread & 0.74 & 0.11 & 0.07 & 0.03 \\
$\quad$ Global degree of coherence, $\zeta_{\mathrm{XRT}}^{\mathrm{DC}}$ & 0.85 & 0.28 & 0.26 & 0.15 \\
$\quad$ Coherent fraction, $\zeta_{\mathrm{XR}}^{\mathrm{CF}}$ & 0.89 & 0.26 & 0.21 & 0.12 \\
$\quad$ Coherent fraction, $\zeta_{\text {Analyt. }}^{\mathrm{CF}}$ & & & & \\
\hline
\end{tabular}

\subsection{Coherent fraction of radiation}

As soon as the mode values are normalized by the sum of all modes $\sum_{j=0}^{\infty} \beta_{j}(\omega)$ (as it is implemented in the XRT software), the value of the first mode naturally gives the coherent fraction of radiation [see equation (13)]. The values of the coherent fraction are presented in Fig. 5 for all photon energies considered in this work (shown by triangles) as a function of electron beam emittance for different values of energy spread. We can see from this figure (see also Table 4) that at $10 \mathrm{pm}$ rad we are getting very high coherence values of about $95 \%$ at $500 \mathrm{eV}$ and $55 \%$ at $12 \mathrm{keV}$ at zero energy spread. With the increase of the energy spread these values become slightly lower at the energy spread values of $1 \times 10^{-3}(91 \%$ and $41 \%)$ and significantly lower at the energy spread values of $2 \times 10^{-3}$ ( $85 \%$ and $28 \%$ ). At higher photon energies the coherent fraction of the radiation drops from $40 \%$ to $26 \%$ at $24 \mathrm{keV}$ and from $26 \%$ to $15 \%$ at $50 \mathrm{keV}$, while energy spread increases from zero to $2 \times 10^{-3}$.

We compared these results of XRT simulations with calculations of coherent fraction in the frame of the analytical approach (shown by circles in Fig. 5). Analytical simulations were performed by taking field amplitudes at the source and in the far-field region according to equations (32)-(33) and performing mode decomposition similar to the $X R T$ code (see the supporting information for details). We see from Fig. 5 that the results of the analytical approach fit well to the results of the $X R T$ simulations for all energies and energy spread values considered in this work.

\subsection{Cross-spectral density function analyzed in one transverse direction}

Finally, we would like to compare the results obtained in the previous sections with the results of simulations of correlation functions in one direction. The coherent-mode representation of correlation functions, being very general, is providing an excellent theoretical insight into the problem. At the same 

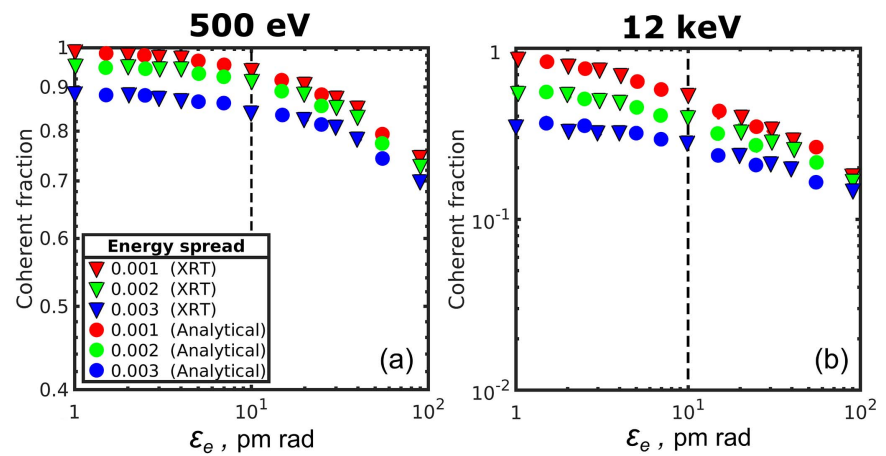

$24 \mathrm{keV}$

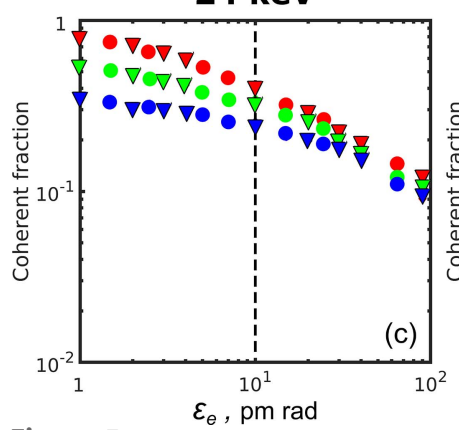

Figure 5

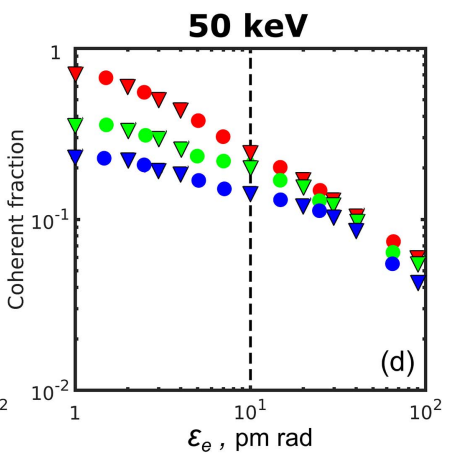

Coherent fraction of radiation $\zeta^{\mathrm{CF}}$ as a function of the electron beam emittance $\varepsilon_{\mathrm{e}}$ for the different values of the photon energy and energy spread in one transverse direction. Triangles are $X R T$ simulations, circles are analytical calculations performed according to equation (13). Red, green and blue colour correspond to $0,1 \times 10^{-3}$ and $2 \times 10^{-3}$ relative energy spread values, respectively. Dashed vertical line corresponds to the value of the electron emittance of $10 \mathrm{pm}$ rad. Note the different scale for $500 \mathrm{eV}$ coherent fraction value.

time, the shape of the coherent modes is not easy to determine experimentally. Since the cross-spectral density $W\left(\mathbf{r}_{1}, \mathbf{r}_{2}\right)$ is a four-dimensional function, its determination from the experimental data is not a simple task. In most of the experiments (such as Young's double pinhole experiment), correlation functions are determined in each direction separately (see, for example, Vartanyants et al., 2011; Singer et al., 2012; Skopintsev et al., 2014). Next, it is assumed that the CSD of the whole field may be represented as a product of its orthogonal directions [see equation (17)]. We analysed this question for the case of the diffraction-limited source.

Simulations of the correlation functions in one transverse direction were performed in the far-field region at $30 \mathrm{~m}$ distance from the source. To determine these functions the $X R T$ software and analytical expression of the wavefield from a single electron in the far-field region [see equation (32)] were used. The results of these simulations for two photon energies of $500 \mathrm{eV}$ and $12 \mathrm{keV}$ are presented in Table 5 and Fig. 6 (results of the analytical approach for the same energies are shown in Figs. S5-S6 of the supporting information). The intensity distribution $I(x, y)$ [Figs. $6(a)$ and $6(b)$ ], absolute value of the CSD in the horizontal direction $\left|W\left(x_{1}, x_{2}\right)\right|$ [Figs. 6(c) and 6(d)], absolute value of the SDC $\left|\mu\left(x_{1}, x_{2}\right)\right|$ [Figs. 6(e) and 6(f)], and absolute value of the spectral degree of coherence as a function of spatial separation of two points $|\mu(\Delta x)|[$ Figs. $6(g)$ and $6(h)]$ for both energies are presented

Table 5

Degree of coherence in one transverse direction obtained from the XRT simulations at $10 \mathrm{pm}$ rad electron beam emittance compared with the analytical analysis for the different photon energies and relative energy spread values.

\begin{tabular}{lllll}
\hline Photon energy $(\mathrm{keV})$ & 0.5 & 12 & 24 & 50 \\
\hline $\begin{array}{l}\text { Zero relative energy spread } \\
\text { Degree of coherence, } \zeta_{x}^{\text {XRT }}\end{array}$ & 0.92 & 0.37 & 0.26 & 0.15 \\
$\quad$ Degree of coherence, $\zeta_{x}^{\text {Analyt. }}$ & 0.92 & 0.39 & 0.25 & 0.15 \\
Degree of coherence, $\zeta_{x}^{\text {GSM }}$ & 0.87 & 0.39 & 0.28 & 0.16 \\
& & & & \\
$1 \times 10^{-3}$ relative energy spread & & & & \\
Degree of coherence, $\zeta_{x}^{\text {XT }}$ & 0.89 & 0.25 & 0.17 & 0.11 \\
Degree of coherence, $\zeta_{x}^{\text {Analyt. }}$ & 0.89 & 0.23 & 0.17 & 0.09 \\
Degree of coherence, $\zeta_{x}^{\text {GSM }}$ & 0.85 & 0.29 & 0.19 & 0.11 \\
& & & & \\
$2 \times 10^{-3}$ relative energy spread & & & & \\
Degree of coherence, $\zeta_{x}^{\text {XRT }}$ & 0.82 & 0.17 & 0.12 & 0.07 \\
Degree of coherence, $\zeta_{x}^{\text {Analyt. }}$ & 0.80 & 0.15 & 0.12 & 0.07 \\
Degree of coherence, $\zeta_{x}^{\text {GSM }}$ & 0.84 & 0.21 & 0.14 & 0.09 \\
\hline
\end{tabular}

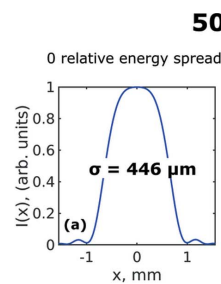

$500 \mathrm{eV}$

$10^{-3}$ relative energy spread
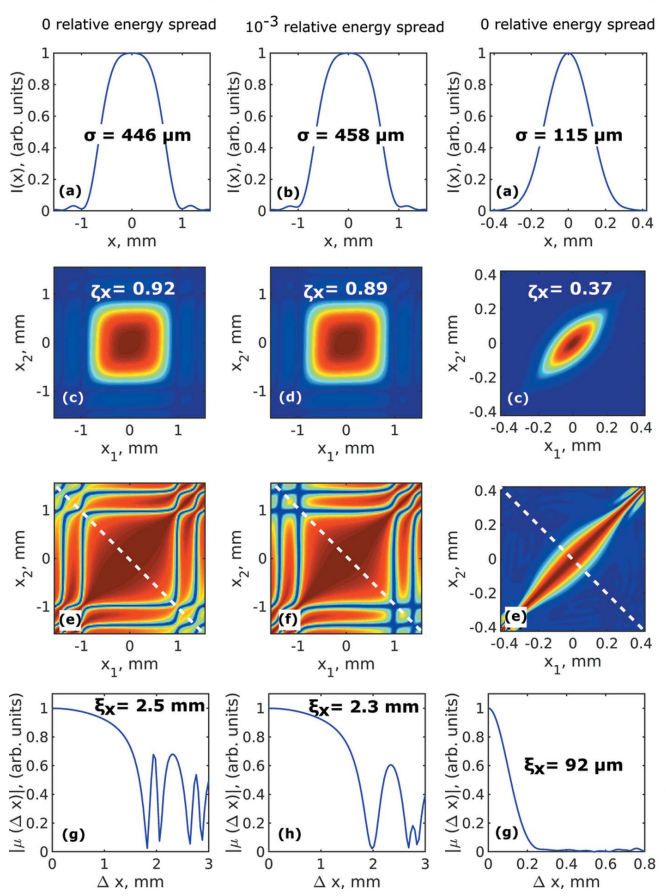

$12 \mathrm{keV}$

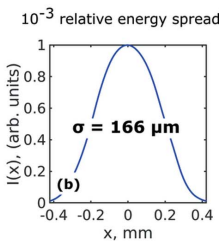

Figure 6

Simulations of the correlation functions in the horizontal direction performed by the XRT software for $500 \mathrm{eV}$ (left column) and $12 \mathrm{keV}$ (right column) photon energy. Intensity distribution $I(x)(a, b)$, absolute value of the cross-spectral density in the horizontal direction $\left|W\left(x_{1}, x_{2}\right)\right|$ $(c, d)$, absolute value of the $\operatorname{SDC}\left|\mu\left(x_{1}, x_{2}\right)\right|(e, f)$, and absolute value of the spectral degree of coherence along the anti-diagonal line [shown in $(e, f)]$ as a function of separation of two points $|\mu(\Delta x)|(g, h)$ simulated in the horizontal direction $30 \mathrm{~m}$ downstream from the undulator source. In $(a, b) \sigma$ is the r.m.s. value of the beam size, in $(c, d) \zeta_{x}$ is the transverse degree of coherence, and in $(g, h) \xi_{x}$ is the coherence length determined in the horizontal direction.

in Fig. 6. It is easy to see from this figure that the functional dependence of these parameters is non-Gaussian for the $500 \mathrm{eV}$ photon energy. But already at $12 \mathrm{keV}$ these parameters can be safely described by Gaussian functions (the same is valid for higher energies). As a general rule, the more 
modes contribute to the CSD, the more this dependence resembles Gaussian. We determined the root mean square (r.m.s.) values $\sigma_{x, y}$ of the intensity distribution in the far-field region using equations (34) [see Figs. 6(a) and 6(b)]. The values of the transverse degree of coherence $\zeta_{x}^{\mathrm{DC}}$ [see Figs. 6(c) and $6(d)$ ] were calculated according to equation (5). Antidiagonal cuts of the spectral degree of the coherence function [see Figs. 6(e) and 6(f)] were used to determine the coherence length of radiation $\xi_{x}$ as its r.m.s. values according to equations (34). As can be seen from Fig. 6, results of simulations performed by the $X R T$ software match extremely well to the ones performed analytically (see Figs. S5-S6 of the supporting information).

As can be seen from our simulations, the CSD function $W\left(x_{1}, x_{2}\right)$ has a rectangular shape [see Figs. 6(c) and 6(d)] because the coherence length of the beam is larger than the beam size at $500 \mathrm{eV}$. This is similar to our earlier observations at the X-ray free-electron laser source (Gorobtsov et al., 2018). Also, in this diffraction-limited case, the SDC shows strong oscillations at the tails of the beam profile due to the fact that the total photon radiation is defined mostly by characteristics of single-electron radiation. This is also a reason why a Gaussian approximation is not valid in this diffraction-limited case and more careful analysis is required. Similar to our previous studies we also observed a decrease of the values of the transverse degree of coherence with the increase of energy spread (see Table 5). Similar simulations were performed for higher photon energies of $24 \mathrm{keV}$ and $50 \mathrm{keV}$, and are summarized in Figs. S3-S4 of the supporting information and in Table 5.

Note that although the Gaussian approximation fits nicely for the spectral degree of coherence profile as well as for cross-spectral density at higher energies, the global degree of coherence is not equal to the product of transverse coherence values, $\zeta \neq \zeta_{x} \zeta_{y}$ (compare Tables 4 and 5). This leads to the conclusion that correlation functions for synchrotron radiation close to the diffraction limit cannot be factorized in the two transverse directions.

\section{Conclusions and outlook}

In summary, we have provided a detailed analysis of the coherence properties of a high-energy synchrotron storage ring with ultra-low emittance values near $10 \mathrm{pm} \mathrm{rad}$ and a wide range of photon energies from $500 \mathrm{eV}$ to $50 \mathrm{keV}$. Such low values of electron beam emittance are expected to be reached at the PETRA IV facility (Schroer et al., 2018, 2019). In addition, we analyzed the effect of electron energy spread on radiation properties of a low-emittance synchrotron ring for the same energy range. All simulations were performed using $X R T$ software and were additionally compared with the results of analytical simulations based on equations of synchrotron radiation and also with approximated formulas given by Tanaka \& Kitamura (2009). We note that all three approaches produced similar results for the whole set of parameters investigated in this work.
The following important lessons were learned during this work. In order to determine the properties of radiation from diffraction-limited sources, an approach based on statistical optics (Goodman, 1985; Mandel \& Wolf, 1995) should be used $^{3}$. For low-emittance storage rings, the radiation field can no longer be approximated by Gaussian functions. The single electron radiation distribution defines the beam profile in this low-emittance regime. As a consequence, a minimum photon emittance (diffraction limit) that may be reached on such storage rings is rather $\lambda / 2 \pi$ than $\lambda / 4 \pi$, typical for Gaussian beams. As a result, the degree of coherence of the radiation goes to its asymptotic limit and approaches unity when the photon emittance is reaching the value of $\lambda / 2 \pi$.

Another lesson is that even for such low emittance values as $10 \mathrm{pm}$ rad the true diffraction limit will be reached; in fact, only at soft X-ray energies of about $500 \mathrm{eV}$. In this case, only a few modes will contribute to the radiation field, but already at $12 \mathrm{keV}$ the radiation field will consist of about ten modes and will start to be more Gaussian-like. This effect will be even more pronounced at high energies. In order to reach the true diffraction limit for hard X-rays, the emittance should be pushed down to about $1 \mathrm{pm} \mathrm{rad}$, that is, unfortunately, out of reach for present technology.

Our results also show that correlation functions, describing radiation field properties such as the degree of coherence, cannot be factorized into two transverse directions for these low-emittance sources. For a full description of the radiation properties of these sources, eigenvalue decomposition of the radiation field has to be performed which offers good theoretical insight as well as complete generality. It also means that present experimental approaches which measure coherence properties of radiation in each direction separately (Vartanyants et al., 2011; Singer et al., 2012; Skopintsev et al., 2014) should be generalized to $2 \mathrm{D}$ methods of coherence determination.

Another outcome of our work is the analysis of the electron energy spread effects on coherence properties of low-emittance storage rings. We demonstrate that this effect becomes more noticeable for low electron beam emittance. The larger the energy spread values, the more the source size and divergence are affected, and, as a consequence, the degree of coherence and coherent fraction value of the radiation are decreased. We found that, in order to keep high coherence values of radiation, the relative energy spread should not exceed the value of $1 \times 10^{-3}$ at the electron emittance values of $10 \mathrm{pm} \mathrm{rad}$. We should note that at $1 \mathrm{pm} \mathrm{rad}$ emittance values the energy spread should be sufficiently smaller than $1 \times 10^{-3}$ to keep high coherence of the X-ray beam.

Finally, our results demonstrate that the coherence properties of the future diffraction-limited sources will be outstanding (Hettel, 2014; Weckert, 2015). We hope that the general approach and new tools for an adequate description of the coherence properties of synchrotron sources, provided in

\footnotetext{
${ }^{3}$ As shown in our work, the Gaussian Schell-model, discussed earlier, should be used with caution for diffraction-limited sources.
} 
this work, will be helpful for the design and planning of future diffraction-limited sources worldwide.

\section{Related literature}

The following references, not cited in the main body of the paper, have been cited in the supporting information: Jackson (1962); Sanchez del Rio (2018).

\section{Acknowledgements}

The authors would like to thank E. Weckert for fruitful discussions and support of the project, R. Chernikov and K. Klementiev for the support with the $X R T$ software and discussion of the results, M. Tischer, G. Geloni and Yu. Obukhov for careful reading of the manuscript, and E. Saldin for fruitful discussions.

\section{Funding information}

The following funding is acknowledged: Helmholtz Associations Initiative and Networking Fund and the Russian Science Foundation grant HRSF-0002.

\section{References}

Alferov, D., Bashmakov, Y. \& Bessonov, E. (1973). Zh. Tekhnich. Fiz. 43, 2126-2132.

Chubar, O. \& Elleaume, P. (1998). Proceedings of the Sixth European Particle Accelerator Conference (EPAC'98), 22-26 June 1998, Stockholm, Sweden, pp. 1177-1179.

Coisson, R. (1988). Opt. Eng. 27, 250-252.

Eriksson, M., van der Veen, J. F. \& Quitmann, C. (2014). J. Synchrotron Rad. 21, 837-842.

Geloni, G., Kocharyan, V. \& Saldin, E. (2015). J. Synchrotron Rad. 22, 288-316.

Geloni, G., Saldin, E., Schneidmiller, E. \& Yurkov, M. (2008). Nucl. Instrum. Methods Phys. Res. A, 588, 463-493.

Geloni, G., Serkez, S., Khubbutdinov, R., Kocharyan, V. \& Saldin, E. (2018). J. Synchrotron Rad. 25, 1335-1345.

Goodman, J. W. (1985). Statistical Optics. New York: John Wiley \& Sons.

Gori, F. (1983). Opt. Commun. 46, 149-154.

Gorobtsov, O. Y., Mukharamova, N., Lazarev, S., Chollet, M., Zhu, D., Feng, Y., Kurta, R. P., Meijer, J. M., Williams, G., Sikorski, M., Song, S., Dzhigaev, D., Serkez, S., Singer, A., Petukhov, A. V. \& Vartanyants, I. A. (2018). Sci. Rep. 8, 2219.

Hettel, R. (2014). J. Synchrotron Rad. 21, 843-855.

Jackson, J. D. (1962). Classical Electrodynamics. New York: John Wiley \& Sons.

Kim, K. (1986). Nucl. Instrum. Methods Phys. Res. A, 246, 71-76.

Kim, K. (1989). AIP Conf. Proc. 184, 565-632.

Klementiev, K. \& Chernikov, R. (2014). Proc. SPIE, 9209, 92090A.
Lee, H. J. \& Zhang, Z. (2007). J. Thermophys. Heat Transfer, 21, 330-336.

Mandel, L. \& Wolf, E. (1995). Optical Coherence and Quantum Optics. Cambridge University Press.

Meng, X., Xue, C., Yu, H., Wang, Y., Wu, Y. \& Tai, R. (2015). Opt. Express, 23, 29675-29686.

Onuki, H. \& Elleaume, P. (2003). Undulators, Wigglers and their Applications. London: Taylor \& Francis.

Rodrigues, A. R. D., Arroyo, F. C., Bagnato, O. R., Citadini, J. F., Farias, R. H. A., Franco, J. G. R. S., Junqueira Leao, R., Liu, L., Marques, S. R., Neuenschwander, R. T., Rodrigues, C., Rodrigues, F., Seraphim, R. M. \& Silva, O. H. V. (2018). Proceedings of the 9th International Particle Accelerator Conference (IPAC2018), 29 April-4 May 2018, Vancouver, BC, Canada, pp. 2886-2889. THXGBD4.

Sanchez del Rio, M. (2018). arXiv: 1801.07542v2 [physics.acc-ph].

Schroer, C. G., Agapov, I., Brefeld, W., Brinkmann, R., Chae, Y.-C., Chao, H.-C., Eriksson, M., Keil, J., Nuel Gavaldà, X., Röhlsberger, R., Seeck, O. H., Sprung, M., Tischer, M., Wanzenberg, R. \& Weckert, E. (2018). J. Synchrotron Rad. 25, 1277-1290.

Schroer, C. G. et al. (2019). PETRA IV: Upgrade of PETRA III into the Ultimate $3 D$ Process Microscope, Conceptual Design Report. DESY, Hamburg, Germany.

Schroer, C. G. \& Falkenberg, G. (2014). J. Synchrotron Rad. 21, 9961005.

Shi, X., Reininger, R., Sanchez del Rio, M. \& Assoufid, L. (2014). J. Synchrotron Rad. 21, 669-678.

Shpyrko, O. G. (2014). J. Synchrotron Rad. 21, 1057-1064.

Siegman, A. E. (1986). Lasers. University Science Books.

Singer, A., Sorgenfrei, F., Mancuso, A. P., Gerasimova, N., Yefanov, O. M., Gulden, J., Gorniak, T., Senkbeil, T., Sakdinawat, A., Liu, Y., Attwood, D., Dziarzhytski, S., Mai, D. D., Treusch, R., Weckert, E., Salditt, T., Rosenhahn, A., Wurth, W. \& Vartanyants, I. A. (2012). Opt. Express, 20, 17480-17495.

Singer, A. \& Vartanyants, I. A. (2014). J. Synchrotron Rad. 21, 5-15. Skopintsev, P., Singer, A., Bach, J., Müller, L., Beyersdorff, B., Schleitzer, S., Gorobtsov, O., Shabalin, A., Kurta, R. P., Dzhigaev, D., Yefanov, O. M., Glaser, L., Sakdinawat, A., Grübel, G., Frömter, R., Oepen, H. P., Viefhaus, J. \& Vartanyants, I. A. (2014). J. Synchrotron Rad. 21, 722-728.

Starikov, A. \& Wolf, E. (1982). J. Opt. Soc. Am. 72, 923-928.

Tanaka, T. \& Kitamura, H. (2009). J. Synchrotron Rad. 16, 380-386.

Tavares, P. F., Leemann, S. C., Sjöström, M. \& Andersson, A. (2014). J. Synchrotron Rad. 21, 862-877.

Vartanyants, I. A. \& Singer, A. (2010). New J. Phys. 12, 035004.

Vartanyants, I. A., Singer, A., Mancuso, A. P., Yefanov, O. M., Sakdinawat, A., Liu, Y., Bang, E., Williams, G. J., Cadenazzi, G., Abbey, B., Sinn, H., Attwood, D., Nugent, K. A., Weckert, E., Wang, T., Zhu, D., Wu, B., Graves, C., Scherz, A., Turner, J. J., Schlotter, W. F., Messerschmidt, M., Lüning, J., Acremann, Y., Heimann, P., Mancini, D. C., Joshi, V., Krzywinski, J., Soufli, R., Fernandez-Perea, M., Hau-Riege, S., Peele, A. G., Feng, Y., Krupin, O., Moeller, S. \& Wurth, W. (2011). Phys. Rev. Lett. 107, 144801.

Vartanyants, I. \& Singer, A. (2016). Synchrotron Light Sources and Free-Electron Lasers, pp. 821-863. Springer International.

Walker, R. P. (2019). Phys. Rev. Accel. Beams, 22, 050704.

Weckert, E. (2015). IUCrJ, 2, 230-245.

Wigner, E. (1932). Phys. Rev. 40, 749-759. 